\title{
ON QUASI-PARABOLIC PROBLEMS WITH NORMAL BOUNDARY CONDITIONS
}

\author{
VEIKKO T. PURMONEN
}

\section{Introduction}

In the present paper we study initial-boundary value problems of the form

$$
\begin{array}{lll}
A\left(\partial / \partial t, D_{x}\right) u=f & \text { in } & \boldsymbol{R}_{+} \times \boldsymbol{R}_{+}^{n}, \\
\left.\frac{\partial^{k} u}{\partial t^{k}}\right|_{t=0}=\varphi_{k} & \text { on } & \boldsymbol{R}_{+}^{n}, \\
\left.B_{j}\left(\partial / \partial t, D_{x}\right) u\right|_{x_{n}=0}=g_{j} & \text { on } & \boldsymbol{R}_{+} \times \boldsymbol{R}^{n-1},
\end{array}
$$

where $A\left(\partial / \partial t, D_{x}\right)$ and $B_{j}\left(\partial / \partial t, D_{x}\right)$ are linear partial differential operators with constant complex coefficients or, briefly, differential operators in $\boldsymbol{R}_{t} \times \boldsymbol{R}_{x}^{n}$. The problems considered are quasi-parabolic in the sense defined in [2] and their lateral boundary operator system $\left\{B_{j}\left(\partial / \partial t, D_{x}\right)\right\}$ is normal to the hyperplane $x_{n}=0$.

In our previous work [2] we gave a necessary and sufficient condition for problems of quasi-parabolic type to have solutions in a setting of certain anisotropic Sobolev-type spaces. We also proved a necessary and sufficient condition for the validity of an a priori estimate between a solution and the data. The aim of this paper is to show that the general conditions mentioned above will take very concrete forms in the case under consideration. The special case in which the lateral boundary operators are of the form $B_{j}=B_{j}\left(D_{x}\right)$ was already discussed for illustration in [2]. The general case is, however, more difficult and requires a different treatment.

We state our main results, Theorem 2.3 on the solvability and Theorem 2.4 on the a priori estimate, in Section 2 after the preliminary first section; in order to avoid overlapping in introducing the necessary notions we shall refer to [2]. The proofs of the main results make essential use of some propositions concerning weighted anisotropic Sobolev spaces and of a trace result; which are given in Sections 3 and 4, respectively. In Section 5 we prove Theorem 2.3, and the proof of Theorem 2.4 is finally given in Section 6. Note that throughout the paper the symbol $C$ is used to denote a generic positive constant.

It seems to us that some more or less loose ideas of compatibility appearing in considerations of initial-boundary value problems can be made precise by the methods of [2] and this work. 


\section{Preliminaries}

1.1. We begin with some notation. We set

$$
\langle x, \xi\rangle=x_{1} \xi_{1}+\ldots+x_{n} \xi_{n}
$$

for $x=\left(x^{\prime}, x_{n}\right)=\left(x_{1}, \ldots, x_{n-1}, x_{n}\right) \in \boldsymbol{R}^{n}=\boldsymbol{R}_{x}^{n}$ and $\xi=\left(\xi_{1}, \ldots, \xi_{n}\right) \in \overline{\boldsymbol{R}}^{n}$. Let

$$
\begin{aligned}
& \boldsymbol{R}_{+}^{n}=\left\{x=\left(x^{\prime}, x_{n}\right) \in \boldsymbol{R}^{n} \mid x_{n}>0\right\}, \\
& Q=\boldsymbol{R}_{+} \times \boldsymbol{R}_{+}^{n} \subset \boldsymbol{R}_{t} \times \boldsymbol{R}_{x}^{n}, \\
& \Sigma=\boldsymbol{R}_{+} \times \boldsymbol{R}^{n-1} \subset \boldsymbol{R}_{t} \times \boldsymbol{R}_{x^{\prime}}^{n-1},
\end{aligned}
$$

where

$$
\boldsymbol{R}_{+}=\boldsymbol{R}_{t,+}=\left\{t \in \boldsymbol{R}_{t} \mid t>0\right\} .
$$

For every multi-index $\alpha=\left(\alpha_{1}, \ldots, \alpha_{n}\right) \in N^{n}$ we write

$$
\xi^{\alpha}=\xi_{1}^{\alpha_{1}} \ldots \xi_{n}^{\alpha_{n}} \quad \text { and } \quad D^{\alpha}=D_{1}^{\alpha_{1}} \ldots D_{n}^{\alpha_{n}}
$$

with $D=D_{x}=\left(D_{1}, \ldots, D_{n}\right), D_{k}=-i \partial / \partial x_{k}(i=\sqrt{-1}) ;$ in addition, set $\partial_{t}=\partial / \partial t$. By $\gamma_{t}$ and $\gamma_{n}$ we denote the trace operators with respect to $t$ and $x_{n}$, respectively.

1.2. Let $m_{0}, m_{1}, \ldots, m_{n}$ be positive integers, $\mu=\max \left\{m_{k}\right\}, q_{k}=\mu / m_{k}$, and $q=\left(q_{1}, \ldots, q_{n}\right)$.

We shall consider differential operators

and

$$
A\left(\partial_{t}, D\right)=\sum_{k g_{0}+\langle\alpha, q\rangle \leqq \mu} a_{k \alpha} \partial_{t}^{k} D^{\alpha} \quad\left(k \in N, \alpha \in N^{n}\right)
$$

$$
B_{j}\left(\partial_{t}, D\right)=\sum_{k q_{0}+\langle\alpha, q\rangle \leqq \mu_{j}} b_{j k \alpha} \partial_{t}^{k} D^{\alpha}, \quad j=1, \ldots, \chi,
$$

where $a_{k \alpha}$ and $b_{j k \alpha}$ are complex constants, $\mu_{j} \geqq 0$ is the order of $B_{j}\left(\partial_{t}, D\right)$, and $x \leqq m_{n}$ is a positive integer. We can and shall write them in a self-explanatory manner also in the form

$$
\begin{aligned}
& A\left(\partial_{t}, D\right)=\sum_{k \leqq m_{0}} A_{k}(D) \partial_{t}^{k}, \\
& B_{j}\left(\partial_{t}, D\right)=\sum_{k q_{0} \leqq \mu_{j}} B_{j k}(D) \partial_{t}^{k} ;
\end{aligned}
$$

note that we prefer here to write for example $A_{k}(D)$ instead of $A^{(k)}(D)$ used in [2]•

1.3. Problem (QP). We shall study the initial-boundary value problem

$$
\begin{array}{ll}
A\left(\partial_{t}, D\right) u=f & \text { in } \quad Q, \\
\gamma_{t} \partial_{t}^{k} u=\varphi_{k} & \text { on } \quad \boldsymbol{R}_{+}^{n}, \quad k=0, \ldots, m_{0}-1, \\
\gamma_{n} B_{j}\left(\partial_{t}, D\right) u=g_{j} & \text { on } \quad \Sigma, \quad j=1, \ldots, \varkappa ;
\end{array}
$$

as in [2] we call it Problem $(Q P)$. 
In the realizations of Problem (QP) the data $f, \varphi_{k}, g_{j}$, and the solution $u$ will lie in certain function spaces of the Sobolev type.

1.4. Basic spaces. The norm of a (complex) normed space $X$ is denoted by $\|\cdot\|_{X}$. If $X, Y$, and $Z$ are three normed spaces with $X \subset Z$ and $Y \subset Z$ algebraically and topologically, let $X \cap Y$ be equipped with the norm

$$
\|u\|_{X \cap Y}=\left(\|u\|_{X}^{2}+\|u\|_{Y}^{2}\right)^{1 / 2} .
$$

Let $s \geqq 0$ and let $X$ be a Hilbert space. Assign the weights $q_{0}$ and $q_{k}$ to differentiation with respect to $t$ and $x_{k}$, respectively. Then we can introduce in a usual way the Sobolev-type space $H^{s}(\Omega ; X)$ of $X$-valued distributions $\Omega \rightarrow X$, used here for $\Omega=\boldsymbol{R}$ or $\boldsymbol{R}_{+}$, and further the anisotropic spaces $H^{s}(\Omega)$ with $\Omega=\boldsymbol{R}_{+}^{n}$ or $\boldsymbol{R}^{n-1}$, and $H^{(s)}(\Omega)=H^{s, s}(\Omega), H_{0}^{(s)}(\Omega)$, and $H_{(0)}^{(s)}(\Omega)$ with $\Omega=Q$ or $\Sigma$. For the definitions of these spaces we refer to [2] and to the references given there.

The weighted Sobolev space $H^{s}\left(R_{+} ; \varrho ; X\right)$ with the weight function $e^{-\varrho t}$, $\varrho \geqq 0$, is defined to consist of the distributions $u: \boldsymbol{R}_{+} \rightarrow X$ with $e^{-\varrho t} u \in H^{s}\left(\boldsymbol{R}_{+} ; X\right)$ and is equipped with the norm

Finally, define

$$
\|u\|_{H^{s}\left(R_{+} ; \varrho ; X\right)}=\left\|e^{-\varrho t} u\right\|_{H^{s}\left(R_{+} ; X\right)} .
$$

$$
H^{(s)}(Q ; \varrho)=H^{s}\left(\boldsymbol{R}_{+} ; \varrho ; H^{0}\left(\boldsymbol{R}_{+}^{n}\right)\right) \cap H^{0}\left(\boldsymbol{R}_{+} ; \varrho ; H^{s}\left(\boldsymbol{R}_{+}^{n}\right)\right)
$$

and analogously $H^{(s)}(\Sigma ; \varrho)$ as well as the spaces $H_{0}^{(s)}(\Omega ; \varrho)$ and $H_{(0)}^{(s)}(\Omega ; \varrho)$ with $\Omega=Q$ or $\Sigma$. See [2].

\section{Main results}

Suppose that the operators $A\left(\partial_{t}, D\right)$ and $B_{j}\left(\partial_{t}, D\right), j=1, \ldots, \varkappa$, are given as in 1.2 , and set for brevity

$$
\delta=\frac{1}{2}\left(q_{0}+q_{n}\right)
$$

and

$$
\begin{aligned}
& R\left(\mu_{j}\right)=\left\{s \in R \mid s>\max \left\{\mu_{j}\right\}+q_{n} / 2\right\}, \\
& R\left(\mu, \mu_{j}\right)=\left\{s \in R\left(\mu_{j}\right) \mid s \geqq \mu\right\}, \\
& R_{q_{0}}\left(\mu, \mu_{j}\right)=\left\{s \in R\left(\mu, \mu_{j}\right) \mid s \neq q_{0} / 2 \bmod q_{0}\right\} .
\end{aligned}
$$

Without further mention, we assume $\varrho \geqq 0$.

We make the following hypotheses:

Hypothesis 1. Problem (QP) is quasi-parabolic, i.e., the operator system

$$
\left(A\left(\partial_{t}, D\right),\left(\gamma_{t} \partial_{t}^{k}\right)_{k=0}^{m_{0}}-1,\left(\gamma_{n} B_{j}\left(\partial_{t}, D\right)\right)_{j=1}^{\chi}\right)
$$

is quasi-parabolic in the sense defined in [2]. 
Remark. We can and shall assume that the coefficient $a_{m_{0}, 0}$ is equal to one. Hypothesis 2. The operator system

is normal to $\Sigma$ :

$$
B_{1}\left(\partial_{t}, D\right), \ldots, B_{\varkappa}\left(\partial_{t}, D\right)
$$

(i) $B_{j}\left(\partial_{t}, D\right)$ is normal to $\Sigma$ of order $\mu_{j}, j=1, \ldots, \chi$, that is, $q_{n} \mid \mu_{j}$ and the coefficient of $D_{n}^{\mu_{j} / q_{n}}$ differs from zero;

(ii) $\mu_{j} \neq \mu_{k}$ for $j \neq k$.

2.1. Initial and boundary values. Let $s \in R\left(\mu, \mu_{j}\right)$. Suppose $u \in H^{(s)}(Q ; \varrho)$ and set

$$
\begin{array}{ll}
f=A\left(\partial_{t}, D\right) u, & \\
\varphi_{k}=\gamma_{t} \partial_{t}^{k} u, & k=0, \ldots, m_{0}-1, \\
g_{j}=\gamma_{n} B_{j}\left(\partial_{t}, D\right) u, & j=1, \ldots, \varkappa .
\end{array}
$$

Then, firstly, the initial values

$$
\Phi_{k}=\gamma_{t} \partial_{t}^{k} u, \quad 0 \leqq k q_{0}<s-q_{0} / 2,
$$

satisfy (see [2], Theorem 7.2)

$$
\begin{array}{ll}
\Phi_{k}=\varphi_{k}, & k=0, \ldots, m_{0}-1, \\
\Phi_{m_{0}+r}=T_{r} f+\sum_{v=0}^{m_{0}-1} S_{r-v} \sum_{k=0}^{m_{0}-1-v} A_{k}(D) \varphi_{v+k}, & 0 \leqq r q_{0}<s-\mu-q_{0} / 2 .
\end{array}
$$

Here the operators $S_{r}$ and $T_{r}$ are defined by

and

$$
\begin{array}{ll}
S_{-r}=0, & r=1,2, \ldots, \\
S_{0}=-\mathrm{Id} & (\mathrm{Id}=\text { identity }), \\
S_{r}=-\sum_{k=0}^{m_{0}-1} A_{k}(D) S_{r-m_{0}+k}, & r=1,2, \ldots,
\end{array}
$$

$$
\begin{array}{llrl}
T_{-r}=0, & r & =1,2, \ldots, \\
T_{r}=\gamma_{t} \partial_{t}^{r}-\sum_{k=0}^{m_{0}-1} A_{k}(D) T_{r-m_{0}+k}, & r & =0,1, \ldots
\end{array}
$$

Secondly, the initial values $\Phi_{k}$ and the boundary values $g_{j}$ satisfy the conditions (LCR) and (GCR):

(LCR) If $\mu_{j}+v q_{0}<s-\delta$ with $v \in N$, then

$$
\gamma_{t} \partial_{t}^{v} g_{j}=\gamma_{n} \sum_{k q_{0} \leqq \mu_{j}} B_{j k}(D) \Phi_{v+k} .
$$


(GCR) If $\mu_{j}+v q_{0}=s-\delta$ with $v \in N$, then

$$
\int_{0}^{\infty} \int\left|\sum_{k q_{0} \leqq \mu_{j}}\left(B_{j k}(D) \Phi_{v+k}\right)\left(x^{\prime}, \sigma^{s / q_{0}}\right)-\exp \left(-\varrho \sigma^{s / q_{n}}\right)\left(\partial_{t}^{v} g_{j}\right)\left(\sigma^{s / q_{n}}, x^{\prime}\right)\right|^{2} d x^{\prime} \frac{d \sigma}{\sigma}<\infty .
$$

See [2], § 8 .

If we now set

and define

$$
G^{s}(\varrho)=\prod_{k q_{0}<s-q_{0} / 2} H^{s-k q_{0}-q_{0} / 2}\left(\boldsymbol{R}_{+}^{n}\right) \times \prod_{j=1}^{\varkappa} H^{\left(s-\mu_{j}-q_{n} / 2\right)}(\Sigma ; \varrho)
$$

$$
F^{s}(\varrho)=\left\{V=\left(\left(\Phi_{k}\right)_{k},\left(g_{j}\right)_{j}\right) \in G^{s}(\varrho) \mid \quad V \quad \text { satisfies (LCR) and (GCR) }\right\},
$$

then $F^{s}(\varrho)$ is a Hilbert space with respect to the norm whose square is given by

$$
\begin{aligned}
\left.\|V\|_{F^{s}(\varrho)}^{2}=\|V\|_{G^{s}(\varrho)}^{2}+\left.\sum_{\mu_{j}+v q_{0}=s-\delta} \int_{\mathbf{D}}^{\infty} \int\right|_{k q_{0} \leqq \mu_{j}} \sum_{j k}(D) \Phi_{v+k}\right)\left(x^{\prime}, \sigma^{s / q_{0}}\right) \\
\\
\quad-\left.\exp \left(-\varrho \sigma^{s / q_{n}}\right)\left(\partial_{t}^{v} g_{j}\right)\left(\sigma^{s / q_{n}}, x^{\prime}\right)\right|^{2} d x^{\prime} \frac{d \sigma}{\sigma} .
\end{aligned}
$$

We shall also need the subspace $E^{s}(\varrho)$ of $F^{s}(\varrho)$ which is defined by

with the operator

$$
E^{s}(\varrho)=\Psi\left(H^{(s)}(Q ; \varrho)\right)
$$

See $[2], \S 8$.

$$
\Psi=\left(\left(\gamma_{t} \partial_{t}^{k}\right)_{k},\left(\gamma_{n} B_{j}\left(\partial_{t}, D\right)\right)_{j}\right) .
$$

2.2. We can now state our main results, Theorems 2.3 and 2.4; their proofs will be given in Sections 5 and 6 .

2.3. Theorem. Let $s \in R_{q_{0}}\left(\mu, \mu_{j}\right)$, and let $\varrho>0$ be as in Theorem 10.2 of [2]. Then Problem (QP) with the data

$$
\begin{aligned}
& f \in H^{(s-\mu)}(Q ; \varrho), \\
& \varphi_{k} \in H^{\left(s-k q_{0}-q_{0} / 2\right)}\left(\boldsymbol{R}_{+}^{n}\right), \quad k=0, \ldots, m_{0}-1, \\
& g_{j} \in H^{\left(s-\mu_{j}-q_{n} / 2\right)}(\Sigma ; \varrho), \quad j=1, \ldots, \varkappa,
\end{aligned}
$$

has a solution $u \in H^{(s)}(Q ; \varrho)$ if and only if the conditions (LCR) and (GCR) are satisfied with the $\Phi_{k}$ defined by (1)-(2) in 2.1.

Moreover, there is at most one solution of Problem $(Q P)$.

2.4. Theorem. Let $s$ and $\varrho$ be as in Theorem 2.3. Then, with the notation of 2.1 the a priori estimate

$$
\|u\|_{H^{(s)}(Q ; \varrho)} \leqq C\left(\|f\|_{H^{(s-\mu)}(Q ; \varrho)}+\left\|\left(\left(\Phi_{k}\right)_{k},\left(g_{j}\right)_{j}\right)\right\|_{E^{s}(\varrho)}\right)
$$


or, equivalently (see [2], 12.3),

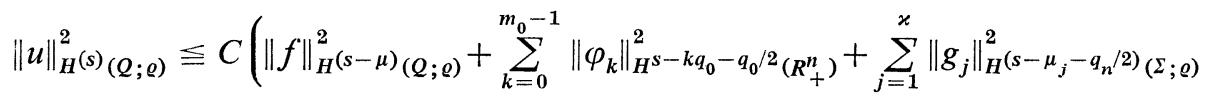

$$
\begin{aligned}
& +\sum_{\mu_{j}+v q_{0}=s-\delta} \int_{0}^{\infty} \int \mid \sum_{k q_{0} \leqq \mu_{j}}\left(B_{j k}(D) \Phi_{v+k}\right)\left(x^{\prime}, \sigma^{s / q_{0}}\right) \\
& \left.-\left.\exp \left(-\varrho \sigma^{s / q_{n}}\right)\left(\partial_{t}^{v} g_{j}\right)\left(\sigma^{s / q_{n}}, x^{\prime}\right)\right|^{2} d x^{\prime} \frac{d \sigma}{\sigma}\right)
\end{aligned}
$$

holds for all $u \in H^{(s)}(Q ; \varrho)$.

Remark. The reversed estimates are valid, too. Note also that the right sides of the estimates virtually depend only on $f,\left(\varphi_{k}\right)_{k}$, and $\left(g_{j}\right)_{j}$.

\section{Some basic results on $H_{(0)}^{(s)}$-spaces}

For the proofs of the main results we shall need the following three propositions. Their proofs will be only briefly sketched.

3.1. Proposition. Let $s \geqq 0$. Then

$$
H_{(0)}^{(s)}(Q ; \varrho)=H^{0}\left(\boldsymbol{R}_{x_{n},+} ; H_{(0)}^{(s)}(\Sigma ; \varrho)\right) \cap H^{s}\left(\boldsymbol{R}_{x_{n},+} ; H^{(0)}(\Sigma ; \varrho)\right)
$$

with equivalent norms.

Proof. We have

Lemma. If $s=k q_{0}$ with $k \in N$, then the spaces $H_{(0)}^{(s)}(Q)$ and

are isomorphic.

$$
H^{0}\left(\boldsymbol{R}_{x_{n},+} ; H_{(0)}^{(s)}(\Sigma)\right) \cap H^{s}\left(\boldsymbol{R}_{x_{n},+} ; H^{(0)}(\Sigma)\right)
$$

In fact, by definition

$$
H_{(0)}^{(s)}(Q)=H_{0}^{s}\left(\boldsymbol{R}_{t,+} ; H^{0}\left(\boldsymbol{R}_{+}^{n}\right)\right) \cap H^{0}\left(\boldsymbol{R}_{t,+} ; H^{s}\left(\boldsymbol{R}_{+}^{n}\right)\right) .
$$

Now use the extension operator and the Fourier transformation with respect to $t$ to show, by Fubini's theorem, that the spaces

and

$$
H_{0}^{k q_{0}}\left(\boldsymbol{R}_{t,+} ; H^{0}\left(\boldsymbol{R}_{+}^{n}\right)\right)
$$

$$
H^{0}\left(\boldsymbol{R}_{x_{n},+} ; H_{0}^{k q_{0}}\left(\boldsymbol{R}_{t,+} ; H^{0}\left(\boldsymbol{R}^{n-1}\right)\right)\right)
$$

are isomorphic. On the other hand, use the extension operator and the Fourier transformation with respect to $x_{n}$ to see that the spaces

$$
H^{0}\left(\boldsymbol{R}_{t,+} ; H^{s}\left(\boldsymbol{R}_{+}^{n}\right)\right)
$$


and

$$
H^{0}\left(\boldsymbol{R}_{x_{n},+} ; H^{0}\left(\boldsymbol{R}_{t,+} ; H^{s}\left(\boldsymbol{R}^{n-1}\right)\right)\right) \cap H^{s}\left(\boldsymbol{R}_{x_{n},+} ; H^{0}\left(\boldsymbol{R}_{t,+} ; H_{r}^{0}\left(\boldsymbol{R}^{n-1}\right)\right)\right)
$$

are isomorphic. Then the assertion follows.

The result of this lemma can be extended by interpolation to all $s \geqq 0$. To complete the proof, apply then the mapping $u \mapsto e^{-\varrho t} u$.

3.2. Proposition. Let $\Omega$ stand for $Q$ or $\Sigma$. If $s_{1}>s_{2} \geqq 0$ and $s_{3}=(1-\theta) s_{1}+$ $\theta s_{2}$ with $0<\theta<1$, then

$$
\left[H_{(0)}^{\left(s_{1}\right)}(\Omega ; \varrho), H_{(0)}^{\left(s_{2}\right)}(\Omega ; \varrho)\right]_{\theta}=H_{(0)}^{\left(s_{3}\right)}(\Omega ; \varrho)
$$

with equivalent norms; here $[X, Y]_{\theta}$ denotes the interpolation space for the interpolation couple $\{X, Y\}$ of two Hilbert spaces $X$ and $Y$ (see [1], Chap. I).

Proof. Since (see [2], Proposition 4.9)

$$
\left[H_{(0)}^{\left(s_{1}\right)}(\Omega), H_{(0)}^{\left(s_{2}\right)}(\Omega)\right]_{\theta}=H_{(0)}^{\left(s_{3}\right)}(\Omega),
$$

it suffices to apply the isomorphism

$$
H_{(0)}^{(s)}(\Omega ; \varrho) \rightarrow H_{(0)}^{(s)}(\Omega): u \mapsto e^{-\varrho t} u .
$$

3.3. Proposition. Let $\Omega$ be as in Proposition 3.2, and let $s \geqq 0$. Then $h \in H_{(0)}^{(s)}(\Omega ; \varrho)$ if and only if

and

(i) $h \in H_{0}^{(s)}(\Omega ; \varrho)$

(ii) $\int_{0}^{\infty} \int\left|\exp (-\varrho \sigma)\left(\partial_{t}^{v} h\right)(\sigma, y)\right|^{2} d y \frac{d \sigma}{\sigma}<\infty \quad$ if $\quad s-q_{0} / 2=v q_{0} \quad$ with $\quad v \in N$.

Condition (i) is equivalent to the condition

(i') $h \in H^{(s)}(\Omega ; \varrho)$ and $\gamma_{t} \partial_{t}^{v} h=0$ for $0 \leqq v q_{0}<s-q_{0} / 2$,

and (ii) to the condition

(ii') $t^{-1 / 2} \partial_{t}^{v} h \in H^{(0)}(\Omega ; \varrho)$.

Furthermore, if $s=v q_{0}+q_{0} / 2$ with $v \in N$, then the square of the norm of $h \in H_{(0)}^{(s)}(\Omega ; \varrho)$ is equivalent to

$$
\|h\|_{H^{(s)}(\Omega ; \varrho)}^{2}+\left\|t^{-1 / 2} \partial_{t}^{v} h\right\|_{H^{(0)}(\Omega ; \varrho)}^{2} .
$$

Proof. In order to prove the first part, it is essentially enough to note that

if and only if $\chi \in H_{0}^{(s)}(\Omega)$ and

$$
\chi=e^{-\varrho t} h \in H_{(0)}^{(s)}(\Omega)
$$

$$
\int_{0}^{\infty} \int\left|\left(\partial_{t}^{v} \chi\right)(\sigma, y)\right|^{2} d y \frac{d \sigma}{\sigma}<\infty \quad \text { if } \quad s=v q_{0}+q_{0} / 2
$$

and then to employ the Leibniz formula. 
To verify the equivalence of the norms, observe first that the square of the norm of $h \in H_{(0)}^{(s)}(\Omega ; \varrho)$ is equivalent to

$$
\left\|e^{-\varrho t} h\right\|_{H^{s}\left(\boldsymbol{R}_{+} ; H^{0}\right)}^{2}+\left\|e^{-\varrho t} h\right\|_{H^{0}\left(\boldsymbol{R}_{+} ; H^{s}\right)}^{2}+\left\|t^{-1 / 2} \partial_{t}^{v}\left(e^{-\varrho t} h\right)\right\|_{H^{0}\left(\boldsymbol{R}_{+} ; H^{0}\right)}^{2},
$$

where $H^{r}$ stands for $H^{r}\left(\boldsymbol{R}_{+}^{n}\right)$ or $H^{r}\left(\boldsymbol{R}^{n-1}\right), r=0, s$. Next show that

$$
\left\|t^{-1 / 2} e^{-\varrho t} \partial_{t}^{k} h\right\|_{H^{0}\left(R_{+} ; H^{0}\right)} \leqq C\|h\|_{H^{(s)}(\Omega ; \varrho)}
$$

for $k<v$, and use the Leibniz formula to obtain

$$
\|h\|_{H_{(0)}^{(s)}(\Omega ; \varrho)}^{2} \leqq C\left(\|h\|_{H^{(s)}(\Omega ; \varrho)}^{2}+\left\|t^{-1 / 2} \partial_{t}^{v} h\right\|_{H^{(0)}(\Omega ; \varrho)}^{2}\right) .
$$

The reversed estimate follows from the inequality

$$
\left\|t^{-1 / 2} \partial_{t}^{v} h\right\|_{H^{(0)}(\Omega ; \varrho)} \leqq C\|h\|_{H_{(0)}^{(s)}(\Omega ; \varrho)},
$$

proved by induction, for example.

\section{A trace result}

4.1. Proposition. Let $s \in R\left(\mu_{j}\right)$. Suppose Hypothesis 2 is satisfied. Then the mapping

$$
\left(\gamma_{n} B_{j}\left(\partial_{t}, D\right)\right)_{j}: H_{(0)}^{(s)}(Q ; \varrho) \rightarrow \prod_{j} H_{(0)}^{\left(s-\mu_{j}-q_{n} / 2\right)}(\Sigma ; \varrho)
$$

is a continuous surjective operator which has a continuous linear right inverse $B_{R}^{-1}$.

Proof. First we have

Lemma. The mapping

$$
\left(\gamma_{n} D_{n}^{j}\right)_{j q_{n}<s-q_{n} / 2}: H_{(0)}^{(s)}(Q ; \varrho) \rightarrow \prod_{j} H_{(0)}^{\left(s-j q_{n}-q_{n} / 2\right)}(\Sigma: \varrho)
$$

is a continuous surjective operator with a continuous linear right inverse $\left(\gamma_{n}^{s}\right)_{R}^{-1}$.

To see this, use a known trace result (see [1], § 4.2), Propositions 3.1 and 3.2, and the open mapping theorem.

From the previous lemma and from Proposition 6.7.4 of [2] it now follows in the usual way (cf. [3], Section 7-8) that the assertion is true for any Dirichlet system

$$
F_{k}\left(\partial_{t}, D\right), \quad 0 \leqq k q_{n}<s-q_{n} / 2,
$$

where the order of $F_{k}\left(\partial_{t}, D\right)$ is $k q_{n}$ and the coefficient of $D_{n}^{k}$ differs from zero. Let $F_{R}^{-1}$ denote the corresponding inverse.

Finally, to prove the general case, it suffices to extend the system $\left\{B_{j}\left(\partial_{t}, D\right)\right\}$ to a Dirichlet system $\left\{F_{k}\left(\partial_{t}, D\right)\right\}$ and then to consider an appropriate restriction of the inverse $F_{R}^{-1}$. 


\section{Proof of the solvability (Theorem 2.3)}

5.1. We first recall the following general result:

Theorem. ([2], Theorem 11.2.) Let $s \in R_{q_{0}}\left(\mu, \mu_{j}\right)$, and let $\varrho$ be as in Theorem 10.2 of [2]. Under Hypothesis 1 Problem $(Q P)$ has a solution $u \in H^{(s)}(Q ; \varrho)$ if and only if

$$
\left(\left(\Phi_{k}\right)_{k},\left(g_{j}\right)_{j}\right) \in E^{s}(\varrho)
$$

Moreover, there is at most one solution.

Thus we are led to prove the next theorem.

5.2. Theorem. Let $s \in R\left(\mu_{j}\right)$. Under Hypothesis 2 we have $E^{s}(\varrho)=F^{s}(\varrho)$.

Proof. Let $V=\left(\left(\Phi_{k}\right)_{k},\left(g_{j}\right)_{j}\right) \in F^{s}(\varrho)$. First define

$$
v=\left(\gamma_{t}^{s}\right)_{R}^{-1}\left(\Phi_{k}\right)_{k} \in H^{(s)}(Q ; \varrho),
$$

where $\left(\gamma_{t}^{s}\right)_{R}^{-1}$ is the continuous linear right inverse of the operator

$$
\gamma_{t}^{s}=\left(\gamma_{t} \partial_{t}^{k}\right)_{k q_{0}<s-q_{0} / 2}: H^{(s)}(Q ; \varrho) \rightarrow \prod_{k} H^{s-k q_{0}-q_{0} / 2}\left(\boldsymbol{R}_{+}^{n}\right) ;
$$

see [2], Proposition 4.7. Set now

and

$$
f_{j}=\gamma_{n} B_{j}\left(\partial_{t}, D\right) v \in H^{\left(s-\mu_{j}-q_{n} / 2\right)}(\Sigma ; \varrho)
$$

Then

$$
h_{j}=g_{j}-f_{j} .
$$

so that

$$
\left(\left(\Phi_{k}\right)_{k},\left(f_{j}\right)_{j}\right)=\Psi v \in E^{s}(\varrho),
$$

Thus we have

$$
\left((0)_{k},\left(h_{j}\right)_{j}\right) \in F^{s}(\varrho) .
$$

and

$$
\gamma_{t} \partial_{t}^{v} h_{j}=0 \quad \text { if } \quad \mu_{j}+v q_{0}<s-\delta
$$

$$
\int_{0}^{\infty} \int\left|\exp (-\varrho \sigma)\left(\partial_{t}^{v} h_{j}\right)\left(\sigma, x^{\prime}\right)\right|^{2} d x^{\prime} \frac{d \sigma}{\sigma}<\infty \quad \text { if } \quad \mu_{j}+v q_{0}=s-\delta .
$$

Hence Proposition 3.3 implies that

$$
h_{j} \in H_{(0)}^{\left(s-\mu_{j}-q_{n} / 2\right)}(\Sigma ; \varrho), \quad j=1, \ldots, \varkappa .
$$

If we set, applying Proposition 4.1,

then

$$
w=B_{R}^{-1}\left(h_{j}\right)_{j} \in H_{(0)}^{(s)}(Q ; \varrho),
$$

$$
\gamma_{n} B_{j}\left(\partial_{t}, D\right) w=h_{j}, \quad j=1, \ldots, \varkappa,
$$


and, by Proposition 3.3,

$$
\gamma_{t} \partial_{t}^{k} w=0 \text { for } 0 \leqq k q_{0}<s-q_{0} / 2 .
$$

Let us now define

Then

$$
u=v+w \in H^{(s)}(Q ; \varrho) .
$$

and

that is,

$$
\gamma_{t} \partial_{t}^{k} u=\Phi_{k}, \quad 0 \leqq k q_{0}<s-q_{0} / 2,
$$

$$
\gamma_{n} B_{j}\left(\partial_{t}, D\right) u=f_{j}+h_{j}=g_{j}, \quad j=1, \ldots, \varkappa,
$$

$$
\left(\left(\Phi_{k}\right)_{k},\left(g_{j}\right)_{j}\right)=\Psi u \in E^{s}(\varrho) .
$$

Thus we have $F^{s}(\varrho) \subset E^{s}(\varrho)$, and consequently $E^{s}(\varrho)=F^{s}(\varrho)$.

\section{Proof of the a priori estimate (Theorem 2.4)}

6.1. We can make use of the next theorem.

Theorem. ([2], Theorem 12.2) Let $s \in R_{q_{0}}\left(\mu, \mu_{j}\right)$, and let $\varrho>0$ be as in Theorem 10.2 of [2]. Under Hypothesis 1 the a priori estimate

$$
\|u\|_{H^{(s)}(Q ; \varrho)} \leqq C\left(\|f\|_{H^{(s-\mu)}(Q ; \varrho)}+\left\|\left(\left(\Phi_{k}\right)_{k},\left(g_{j}\right)_{j}\right)\right\|_{E^{s}(\varrho)}\right)
$$

holds for all $u \in H^{(s)}(Q ; \varrho)$ if and only if the operator

$$
\Psi: H^{(s)}(Q ; \varrho) \rightarrow E^{s}(\varrho)
$$

has a continuous linear right inverse.

Accordingly, it suffices to prove the following result:

6.2. Theorem. Let $s \in R\left(\mu_{j}\right)$. If Hypothesis 2 is satisfied, the operator

$$
\Psi: H^{(s)}(Q ; \varrho) \rightarrow E^{s}(\varrho)
$$

has a continuous linear right inverse.

Proof. We employ the proof of Theorem 5.2, and define a mapping

$$
\Psi_{R}^{-1}: E^{s}(\varrho) \rightarrow H^{(s)}(Q ; \varrho)
$$

by setting, for every $V=\left(\left(\Phi_{k}\right)_{k},\left(g_{j}\right)_{j}\right) \in E^{s}(\varrho)$,

$$
\begin{aligned}
\Psi_{R}^{-1} V & =u=v+w=\left(\gamma_{t}^{s}\right)_{R}^{-1}\left(\Phi_{k}\right)_{k}+B_{R}^{-1}\left(h_{j}\right)_{j} \\
& =\left(\gamma_{t}^{s}\right)_{R}^{-1}\left(\Phi_{k}\right)_{k}+B_{R}^{-1}\left(g_{j}-\gamma_{n} B_{j}\left(\partial_{t}, D\right)\left(\gamma_{t}^{s}\right)_{R}^{-1}\left(\Phi_{k}\right)_{k}\right)_{j} .
\end{aligned}
$$

Then $\Psi_{R}^{-1}$ is a linear right inverse of $\Psi$. 
In order to verify the continuity of $\Psi_{R}^{-1}$, we first write

$$
\left\|\Psi_{R}^{-1} V\right\|_{H^{(s)}(Q ; \varrho)} \leqq\left\|\left(\gamma_{t}^{s}\right)_{R}^{-1}\left(\Phi_{k}\right)_{k}\right\|_{H^{(s)}(Q ; \varrho)}+\left\|B_{R}^{-1}\left(h_{j}\right)_{j}\right\|_{H^{(s)}(Q ; \varrho)} .
$$

Here we have (see [2], Proposition 4.7)

$$
\left\|\left(\gamma_{t}^{s}\right)_{R}^{-1}\left(\Phi_{k}\right)_{k}\right\|_{H^{(s)}(Q ; \varrho)} \leqq \sum_{k}\left\|\Phi_{k}\right\|_{H^{s-k q_{0}-q_{0} / 2}\left(R_{+}^{n}\right)},
$$

and Proposition 4.1 implies that

$$
\left\|B_{R}^{-1}\left(h_{j}\right)_{j}\right\|_{H^{(s)}(Q ; \varrho)} \leqq C \sum_{j}\left\|h_{j}\right\|_{H_{(0)}^{\left(s-\mu_{j}-q_{n} / 2\right)}(\Sigma ; \varrho)},
$$

It follows from Proposition 3.3 that

$$
\begin{aligned}
& \sum_{j}\left\|h_{j}\right\|_{H_{(0)}^{\left(s-\mu_{j}-q_{n} / 2\right)}(\Sigma ; \varrho)}^{2}
\end{aligned}
$$



$$
\begin{aligned}
& =C\left(\Sigma_{1}+\Sigma_{2}\right) \text {. }
\end{aligned}
$$

Using the definition of the $f_{j}$, we obtain here

$$
\Sigma_{1} \leqq C\left(\sum_{j}\left\|g_{j}\right\|_{H_{(0)}^{\left(s-\mu_{j}-q_{n} / 2\right)}(\Sigma ; \varrho)}^{2}+\sum_{k}\left\|\Phi_{k}\right\|_{H^{s-k q_{0}-q_{0} / 2}\left(R_{+}^{n}\right)}^{2}\right) .
$$

In the sum $\Sigma_{2}$ we have

$$
\begin{aligned}
& \left\|t^{-1 / 2} \partial_{t}^{v}\left(g_{j}-f_{j}\right)\right\|_{H^{(0)}(\Sigma ; \varrho)}^{2} \\
& \leqq C\left(\int_{0}^{\infty} \int\left|\sum_{k q_{0} \leqq \mu_{j}}\left(B_{j k}(D) \Phi_{v+k}\right)\left(x^{\prime}, \sigma^{s / q_{0}}\right)-\exp \left(-\varrho \sigma^{s / q_{n}}\right)\left(\partial_{t}^{v} g_{j}\right)\left(\sigma^{s / q_{n}}, x^{\prime}\right)\right|^{2} d x^{\prime} \frac{d \sigma}{\sigma}\right. \\
& \left.\quad+\int_{0}^{\infty} \int\left|\sum_{k q_{0} \leqq \mu_{j}}\left(B_{j k}(D) \Phi_{v+k}\right)\left(x^{\prime}, \sigma^{s / q_{0}}\right)-\exp \left(-\varrho \sigma^{s / q_{n}}\right)\left(\partial_{t}^{v} f_{j}\right)\left(\sigma^{s / q_{n}}, x^{\prime}\right)\right|^{2} d x^{\prime} \frac{d \sigma}{\sigma}\right) .
\end{aligned}
$$

Since $\left(\left(\Phi_{k}\right)_{k},\left(f_{j}\right)_{j}\right)=\Psi v \in E^{s}(\varrho)$, this gives

Thus we have

$$
\Sigma_{2} \leqq C\left(\|V\|_{E^{s}(\varrho)}^{2}+\|v\|_{H^{(s)}(Q ; \varrho)}^{2}\right) \leqq C\|V\|_{E^{s}(\varrho)}^{2} .
$$

$$
\left\|\Psi_{R}^{-1} V\right\|_{H^{(s)}(Q ; \varrho)} \leqq C\|V\|_{E^{s}(\varrho)}
$$

for all $V \in E^{s}(\varrho)$, which completes the proof.

Remark. Note that the existence of a continuous linear right inverse can also be deduced by Theorem 5.2 and the open mapping theorem. 


\section{References}

[1] LiONS, J. L., and E. MAGENES: Non-homogeneous boundary value problems and applications. Volume I. - Die Grundlehren der mathematischen Wissenschaften 181. SpringerVerlag, Berlin-Heidelberg-New York, 1972.

[2] Purmonen, V. T.: Quasi-parabolic initial-boundary value problems. - Ann. Acad. Sci. Fenn. Ser. A I Math. 6, 1981, 29-62.

[3] Schechter, M.: Modern methods in partial differential equations. - McGraw-Hill Inc., 1977.

University of Jyväskylä

Department of Mathematics

Seminaarinkatu 15

SF-40100 Jyväskylä 10

Finland

Received 24 March 1982 